\title{
Aspergillus fumigatus enhances human NK cell activity by regulating M1 macrophage polarization
}

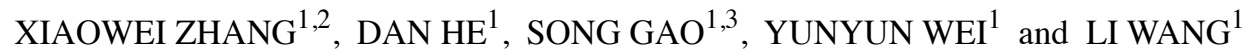 \\ ${ }^{1}$ Department of Pathogenobiology, Jilin University Mycology Research Center, College of Basic Medical Sciences, Jilin University, \\ Changchun, Jilin 130021; ${ }^{2}$ Department of Clinical Laboratory, Changchun Children's Hospital, Changchun, Jilin 130061; \\ ${ }^{3}$ Beijing Zhong Kai Tian Cheng Biotechnology Co., Ltd., Beijing 100176, P.R. China
}

Received October 17, 2018; Accepted April 24, 2019

DOI: $10.3892 / \mathrm{mmr} .2019 .10365$

\begin{abstract}
The progression of disease caused by fungal infection is closely associated with the human immune system. Macrophages and natural killer cells (NK cells) are two important types of innate immune cells that serve an important role in anti-infection immunity. There has been limited research into the interactions between fungi and macrophages. In the present in vitro study, reverse transcription-quantitative PCR, ELISA and flow cytometry were performed to reveal that the interaction between macrophages and NK cells, regulated by Aspergillus fumigatus conidia, induced macrophages to polarize into M1 macrophages by secreting large quantities of tumor necrosis factor- $\alpha$, interleukin-18 and Galectin-9. In addition, when NK cells were co-cultured with the conidia of A.fumigatus-stimulated M1 macrophages, they exhibited increased activation levels and secretion of interferon- $\gamma$ (IFN- $\gamma)$. It was further demonstrated via antibody neutralization and gene silencing experiments that galectin- 9 served an important role in the interaction between macrophages and NK cells regulated by A.fumigatus. In conclusion, it was demonstrated that A. fumigatus induced the polarization of macrophages into M1 macrophages by secreting Galectin-9, which then promoted NK cell activity and IFN- $\gamma$ secretion. The results provided improved understanding of the role of innate immune cells in invasive fungal infections. The present study also provided novel insight into the study of macrophages and NK cells in inflammatory infections caused by A.fumigatus and potential strategies to control the progression of inflammation.
\end{abstract}

Correspondence to: Professor Li Wang, Department of Pathogenobiology, Jilin University Mycology Research Center, College of Basic Medical Sciences, Jilin University, 126 Xinmin Street, Changchun, Jilin 130021, P.R. China

E-mail: wli99@jlu.edu.cn

Key words: Aspergillus fumigatus, fungal infection, macrophage, natural killer cells, galectin-9

\section{Introduction}

Aspergillus fumigatus (A. fumigatus) is an environmentally ubiquitous, spore-forming mould saprophyte that causes disease in individuals with poor immunity (1). The incidence of invasive fungal infections is increased in patients receiving bone marrow and organ transplantation, chemotherapy for cancer, or treatment with glucocorticoids or broad-spectrum use of antibiotics (2). Aspergillosis is the second most common type of fungal infection following candidiasis (3). The use of antifungal agents improves the prognosis of patients to a certain extent; however, the mortality rate of invasive aspergillosis remains high due to the limited efficacy of currently available drugs (4). Infection-mediated immune injury is an important cause of death (5). Immunological diagnosis, prevention and reconstitution are crucial to improving the prognosis of patient recovery (6). Improved understanding of the regulatory mechanisms underlying the host immune response following invasive aspergillosis may aid in improving the prognosis of patients.

Alveolar macrophages serve an important role in innate immunity in the lung tissue, as they exhibit the capacity to identify, process and present antigens, inducing specific immune responses, and are known as an outpost of natural immunity (7). They also serve a role in immune homeostasis via negative feedback regulation of the local microenvironment, preventing excessive immune damage and maintaining the stability of the internal environment (8). A previous study reported that A.fumigatus enhanced the expression of dectin-2 and cluster of differentiation (CD)206 in stimulated macrophages, indicating that the fungus induces a certain regulatory effect on the phenotype and function of macrophages (9).

Together, natural killer cells (NK cells) and macrophages build the first immune defence line in the body for the removal of invasive microbes $(10,11)$. The activation or inhibition of $\mathrm{NK}$ cells is mainly regulated by the expression of activating or inhibitory receptors on the cell surface (12). When NK cells are activated, a large number of cytokines and chemokines are secreted, such as interferon- $\gamma$ (IFN- $\gamma$ ) and tumor necrosis factor- $\alpha$ (TNF- $\alpha)$, which regulate the immune state of the host (13). NK cells have a direct killing effect on invasive pathogens, including bacteria, parasites and yeast (14-16). The effects of NK cells and IFN- $\gamma$ on A. fumigatus were 
first reported in mice (17). The enrichment of NK cells was an important mechanism to prevent further infection of A. fumigatus in a mouse model of neutropenia. Additionally, clinical studies have also demonstrated that IFN- $\gamma$ serves an important role in inhibiting A. fumigatus (18).

The interaction between macrophages and NK cells has attracted widespread attention, particularly in the study of tumor occurrence and development, and microbial invasion and infection. Macrophages and NK cells infiltrate numerous infected or cancerous tissues (19). Monocytes/macrophages serve an important role in immune surveillance and immunoregulation depending on their functions in phagocytosis and antigen presentation (20). Peripheral monocytes frequently differentiate into different subtypes of macrophages depending on the tissue microenvironment (21). The Type 1 T helper (Th1) cytokine IFN- $\gamma$ and lipopolysaccharide (LPS), the ligand of Toll-like receptor 4, polarize monocytes towards a classically activated (M1) macrophage phenotype; M1 macrophages produce proinflammatory cytokines, such as TNF- $\alpha$ and interleukin (IL)-12, subsequently facilitating the clearance of pathogens (22). Conversely, following exposure to Type 2 T helper cytokines, such as IL-4 and IL-13, monocytes differentiate into alternatively activated macrophages (M2 macrophages) inducing the production of anti-inflammatory mediators, including IL-10, which promote anti-inflammation and wound healing (23). Macrophages are affected by phagocytic antigens and the immune microenvironment, express specific receptors or ligands, and secrete large quantities of cytokines and chemokines that regulate NK cell function (24).

Galectin-9, a $\beta$-galactoside binding lectin, is present in various tissues and is particularly abundant in the liver (25). It is a type of eosinophil chemoattractant and is a member of the galactose agglutinin family. Galectin- 9 possesses a variety of biological functions, including contributions to cell differentiation, maturation, adhesion, chemotaxis and apoptosis. It also serves important roles in chronic inflammation, acute inflammation, allergy and autoimmune diseases (26). A recent study reported that macrophages regulated the function of $\mathrm{NK}$ cells by secreting Galectin-9 following hepatitis $\mathrm{C}$ infection (27).

The present study investigated the regulatory effects of Galectin-9 on the interaction between macrophages and NK cells in aspergillosis. It suggested novel directions for further study into the mechanisms underlying the roles of macrophages and NK cells in inflammatory infection caused by A.fumigatus and potential strategies to control the progression of inflammation.

\section{Materials and methods}

Strains of A.fumigatus, activation of spores and culture conditions. The A.fumigatus strain was obtained from the Medical Mycology Research Center of Chiba University. A. fumigatus resting conidia were cultivated for 3 days on beer mash plates at $28^{\circ} \mathrm{C}$. The conidia were detached from the plate using endotoxin-free sterile water and filtered through a cell strainer with a 40- $\mu \mathrm{m}$ nylon mesh pore membrane to obtain a single-fungal cell suspension. The swelling and synchronization of fungal growth was achieved by cultivating the conidia in SDA culture medium (cat. no. HB0235-10; Qingdao Hope Bio-Technology Co., Ltd.) at room temperature under continuous agitation at
200 RPM overnight. The spore morphology was imaged by an Olympus microscope (magnification, x200; CKX41-A32PH; Olympus Corporation). The plates and dishes were obtained from Corning Inc.

Acquisition of macrophages. Peripheral blood mononuclear cells (PBMCs) were freshly isolated from the peripheral blood of 3 healthy individuals at the Changchun Blood Center between May 2017 and September 2017. The 3 donors were all male (24-32 years old). The donors were all negative for hepatitis $\mathrm{B}$, hepatitis $\mathrm{C}$ and human immunodeficiency virus infection. PBMCs were isolated via Ficoll density gradient separation at $500 \mathrm{x} \mathrm{g}$ for $30 \mathrm{~min}$ at room temperature. The study was approved by the Ethics Committee of Changchun Blood Center. All experiments were conducted in accordance with the approved guidelines and regulations (24). Written informed consent for the use of haemocytes in research was obtained from all participants. Monocytes were then purified by magnetic-activated cell sorting with $\mathrm{CD}_{1} 4^{+}$microbeads (Miltenyi Biotec, Inc.) with $3 \times 3 \mathrm{ml}$ of buffer flow through the column (Miltenyi Biotec, Inc.) under the action of gravity. The purity of the cells was $\geq 95 \%$ as determined by flow cytometry. Monocytes were seeded in 96-well plates at a density of $0.2 \times 10^{6}$ cells/well in full RPMI-1640 medium (Thermo Fisher Scientific, Inc.), with $1 \%$ penicillin and streptomycin and $10 \%$ FBS (all from Gibco; Thermo Fisher Scientific, Inc.). Then, $400 \mathrm{IU} / \mathrm{ml}$ granulocyte-macrophage colony-stimulating factor (GM-CSF; cat. no. 300-03-100; PeproTech, Inc.) was added to the culture medium, and the cells were grown at $37^{\circ} \mathrm{C}$ in a $5 \%$ $\mathrm{CO}_{2}$ incubator for 5 days of culture.

Macrophage stimulation experiment. Macrophages were collected following digestion by $0.2 \%$ EDTA at $37^{\circ} \mathrm{C}$ for 5 min (cat. no. 02-032-1ACS; Biological Industries). The cells were reseeded into 12 -well plates at a density of $0.2 \times 10^{6}$ cells/well following three washes with warm PBS buffer (cat. no. 02-024-1ACS; Biological Industries). Swelling conidia were added to the macrophage culture plates, with a ratio of macrophages to conidia of 1:5. Treatment with full RPMI-1640 medium (as above) was used as the control group. Cells and culture supernatant were collected for subsequent experiments following co-culture at $37^{\circ} \mathrm{C}$ for $24 \mathrm{~h}$.

NK cell stimulation experiment. PBMCs were freshly isolated from the peripheral blood of healthy individuals as aforementioned. NK cells were then purified by MACS using an NK Cell Isolation kit (cat. no. 130-092-657; Miltenyi Biotec, Inc.) according to the manufacturer's protocols and were seeded into 12 -well plates at a density of $0.2 \times 10^{6}$ cells/well. Swelling conidia were added to NK cell culture plates, with a ratio of NK cells to conidia of 1:5. Treatment with full RPMI-1640 medium was used as the control group. Cells and culture supernatant were collected for subsequent experiments following co-culture at $37^{\circ} \mathrm{C}$ for $24 \mathrm{~h}$.

Macrophage and NK cell co-culture. Macrophages were collected following digestion by $0.2 \%$ EDTA at $37^{\circ} \mathrm{C}$ for $5 \mathrm{~min}$ and were reseeded into 12 -well plates at a density of $0.2 \times 10^{6}$ cells/well following three washes with warm PBS buffer. An equal quantity of autologous purified NK cells was added 
Table I. Primer sequences for reverse transcription-quantitative PCR.

\begin{tabular}{lll}
\hline Gene & \multicolumn{1}{c}{ Forward primer $\left(5^{\prime}-3 '\right)$} & Reverse primer $\left(5^{\prime}-3^{\prime}\right)$ \\
\hline GAPDH & CGGATTTGGTCGTATTGGG & TCTCGCTCCTGGAAGATGG \\
TNF- $\alpha$ & ATCCTGGGGGACCCAATGTA & AAAAGAAGGCACAGAGGCCA \\
iNOS & GAGGAGCAGGTCGAGGACTAT & TCTTCGCCTCGTAAGGAAATAC \\
CD80 & AAAAGACAGCTACGTGGGTGA & GCCATGTTCTATCGGGTACTTC \\
CD86 & TAGGGCTCCGGATATCTTTG & TCTTGAGGGTCCTTTC TCCA \\
CD163 & TAGGGCTCCGCTTTGGATAT & TCTTGAGGGTCTCCACCTTT \\
CD206 & CAAATCCACGATCAAACCTGTG & AGAACCCTTCATAAGACCACC \\
IL-10 & GGGAGAACCTGAAGACCCTCA & TGCTCTTGTTTCACAGGGAAG
\end{tabular}

$\mathrm{CD}$, cluster of differentiation; iNOS, inducible nitric oxide synthase; TNF- $\alpha$, tumor necrosis factor- $\alpha$.

to the plates, which were then were mixed softly. Swelling conidia were added to the macrophage/NK cell co-culture plates, with a ratio of macrophages to conidia of 1:5. To investigate the contact dependence of the interaction, macrophages and NK cells were separated by a membrane $(0.4-\mu \mathrm{m}$ pore size) in Transwell plates (cat. no. CLS3464-48EA; Costar; Corning Inc.). The swelling conidia were able to contact the macrophages but not the NK cells. Macrophages with conidia or NK cells with conidia were used as controls. The cells and culture supernatant were collected for subsequent experiments following co-culture at $37^{\circ} \mathrm{C}$ for $24 \mathrm{~h}$. In the antibody neutralization test, macrophages were stimulated by A. fumigatus conidia (1:5) for $24 \mathrm{~h}$ and then co-cultured with the NK cells from the same individual for a further $12 \mathrm{~h}$, in the presence or absence of Galectin-9 (cat. no. AF2045; R\&D Systems, Inc.)-, IL-18 (cat. no. D044-3; R\&D Systems, Inc.)- or TNF- $\alpha$ (cat. no. AF-410-NA; R\&D Systems, Inc.)-specific antibodies when NK cells were added. The antibody working concentrations were all $10 \mu \mathrm{g} / \mathrm{ml}$.

Enzyme-linked immunosorbent assay (ELISA). Cell culture supernatants were collected following $24 \mathrm{~h}$ of incubation in each experimental condition. The concentrations of TNF- $\alpha$ (cat. no. BMS223-4; Invitrogen; Thermo Fisher Scientific, Inc.), IL-18 (cat. no. BMS267-2; Invitrogen; Thermo Fisher Scientific, Inc.), Galectin-9 (cat. no. DGAL90; R\&D Systems, Inc.), IL-12 (cat. no. BMS238; Invitrogen; Thermo Fisher Scientific, Inc.) and IL-10 (cat. no. BMS215-2; Invitrogen; Thermo Fisher Scientific, Inc.) were measured by ELISA. The ELISA experiments were performed according to the manufacturer's protocols. The absorbance was detected using a Synergy ${ }^{\mathrm{TM}}$ H1 Full Function Enzyme Marker (BioTek Instruments, Inc.).

Reverse transcription-quantitative PCR (RT-qPCR). RT-qPCR analysis was performed using standard procedures. Total RNA was isolated using a RNeasy kit (Qiagen, Inc.), $5 \mu \mathrm{g}$ of each sample was reverse-transcribed using the M-MLV first-stand synthesis system (Invitrogen; Thermo Fisher Scientific, Inc.) and cDNAs were analyzed in triplicate with the MJ Real-Time PCR System (Bio-Rad Laboratories, Inc.). The sequences of the gene-specific and GAPDH primers used for qPCR are presented in Table I. Amplification was performed for 40 cycles with a denaturation temperature of $94^{\circ} \mathrm{C}$ for $30 \mathrm{sec}$, annealing temperature of $58^{\circ} \mathrm{C}$ for $30 \mathrm{sec}$ and extension temperature of $74^{\circ} \mathrm{C}$ for $45 \mathrm{sec}$ in a Veriti thermal cycler (Applied Biosystems; Thermo Fisher Scientific, Inc.). The PCR products were $200 \mathrm{bp}$ in length. RT-qPCR was performed using the Power SYBR Green Master Mix (Takara Bio, Inc.) and an ABI 7300 Real-Time PCR system (Applied Biosystems, Thermo Fisher Scientific, Inc.). All primers were synthesized by Invitrogen (Thermo Fisher Scientific, Inc.). Fold changes in expression of each gene were calculated by a comparative threshold cycle (Cq) method using the formula $2^{-\Delta \Delta \mathrm{Cq}}(28)$. Data were collected from three independent experiments.

Flow cytometry. Cultured macrophages and/or NK cells were resuspended in staining buffer (1\% FBS in PBS) and were preincubated with $\mathrm{Fc}$ receptor blocking reagent (cat. no. 130-059-901; Miltenyi Biotec, Inc.) for $15 \mathrm{~min}$ at $4^{\circ} \mathrm{C}$. Macrophages were stained with phycoerythrin-conjugated mouse anti-human CD80 (cat. no. 560925; BD Biosciences) and FITC-conjugated mouse anti-human CD86 (cat. no. 560958; BD Biosciences). NK cells were stained with allophycocyanin-conjugated mouse anti-human CD69 (cat. no. 560967; BD Biosciences) on the cell surface. All the antibody were 1:100 dilutions, and incubated in $4^{\circ} \mathrm{C}$ for $15 \mathrm{~min}$. The detection of BUV395-conjugated IFN- $\gamma$ (cat. no. 565971; BD Biosciences) expression in NK cells was performed following cell surface staining. The IFN- $\gamma$ antibody was diluted 1:100 and incubated in $4^{\circ} \mathrm{C}$ for $30 \mathrm{~min}$. Brefeldin A (Sigma-Aldrich; Merck KGaA) was subsequently added for a final concentration of $5 \mu \mathrm{g} / \mathrm{ml}$. A BD LSRIIFortesa ${ }^{\mathrm{TM}}$ flow cytometer (BD Biosciences) was used to perform the experiments, and the acquired data were analyzed with FlowJo version 10 (FlowJo LLC).

RNA interference experiment. Monocytes were purified as aforementioned. The monocytes were seeded into 96 -well plates at a density of $0.2 \times 10^{6}$ cells/well in full RPMI-1640 medium with $400 \mathrm{IU} / \mathrm{ml}$ GM-CSF. The cells were cultured as $37^{\circ} \mathrm{C}$ in a $5 \% \mathrm{CO}_{2}$ incubator for 5 days. Galectin-9-specific small interfering RNA (siRNA) reagent (Sense: 5'GUGCAG AGCUCAGAUUUCATT-3' Antisense: 5'-UGAAAUCUG AGCUCUGCACTT-3', Shanghai GenePharma Co., Ltd.) and the scramble control (Sense: 5'-GCUCAGAUUUCATTG UGCAGA-3' Antisense: 5'-UGAGCUCUGCACTUGAAA 
A

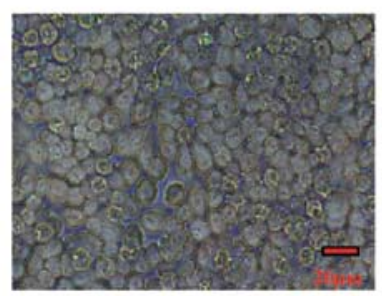

Medium

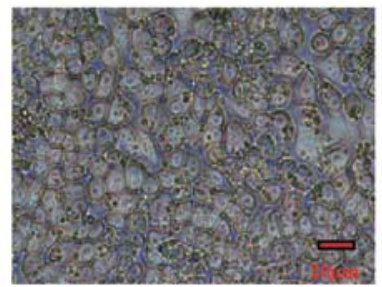

A. fumigatus
B
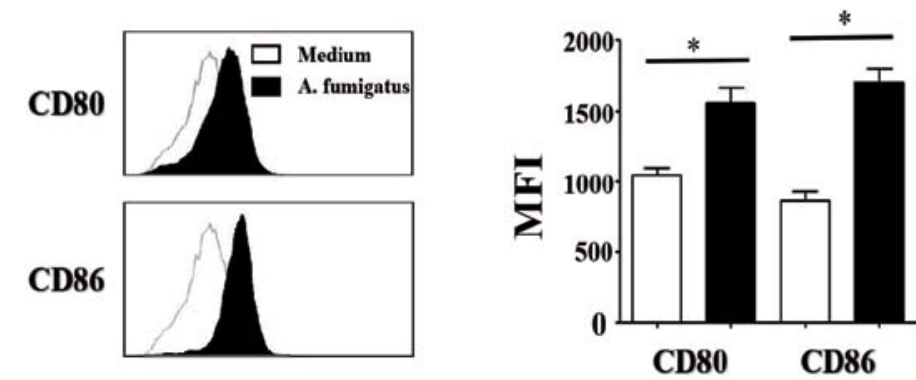

C

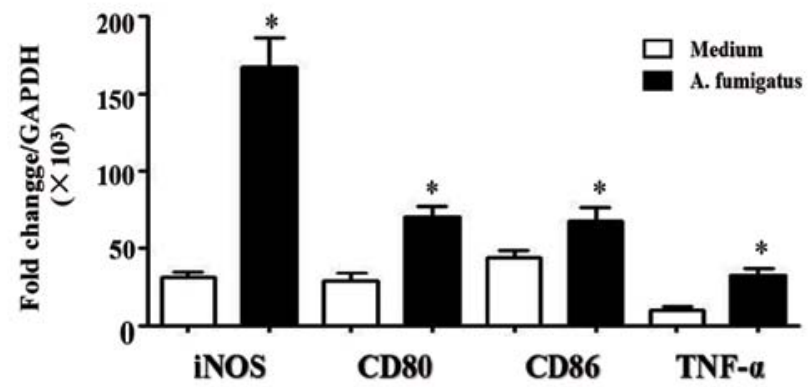

Figure 1. A.fumigatus induces macrophages toward M1 polarization. Monocytes purified from the peripheral blood mononuclear cells of healthy individuals were cultured with granulocyte-macrophage colony-stimulating factor (50 ng/ml) to induce macrophages for 5 days. Conidia of A. fumigatus were obtained following a 5 -h incubation at $37^{\circ} \mathrm{C}$ in an agitator. The macrophages were reseeded into 6 -well plates, stimulated using the conidia (1:5) for $24 \mathrm{~h}$, and then evaluated. (A) Morphology of the macrophages cultured with conidia or control medium. Scale bars, $20 \mu \mathrm{m}$. (B) CD80 and CD86 expression on macrophages and analysis of the MFI ( $\mathrm{n}=3) .{ }^{*} \mathrm{P}<0.05$, as indicated. (C) M1-specific gene expression analysis $(\mathrm{n}=3)$. ${ }^{*} \mathrm{P}<0.05$ vs. Medium. CD, cluster of differentiation; iNOS, inducible nitric oxide synthase; TNF- $\alpha$, tumor necrosis factor- $\alpha$; A. fumigatus, Aspergillus fumigatus; MFI, median fluorescence intensity.

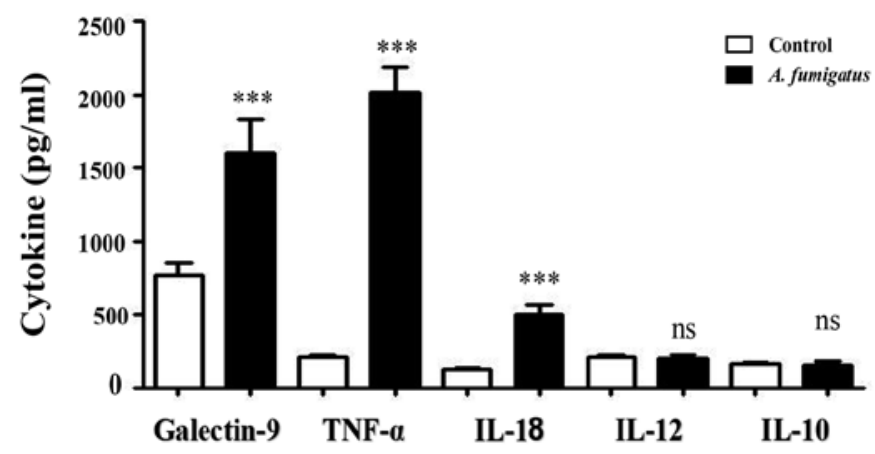

Figure 2. A. fumigatus induces M1 macrophages that secrete Galectin-9, IL-18 and TNF- $\alpha$. Macrophages were treated with A.fumigatus conidia, and the supernatants were collected and evaluated by $\operatorname{ELISA}(\mathrm{n}=3) .{ }^{* * *} \mathrm{P}<0.001$ vs. Control. IL, interleukin; ns, not significant; TNF- $\alpha$, tumor necrosis factor- $\alpha$; A. fumigatus, Aspergillus fumigatus.

UCT 3', Shanghai GenePharma Co., Ltd.) was added to the plates. The cells were transfected with $50 \mathrm{nM}$ of siRNA in Lipofectamine ${ }^{\circledR} 2000$ (Invitrogen; Thermo Fisher Scientific, Inc.). GFP was transfected together with the siRNA treatment to determine transfection efficiency. Galectin-9 expression was detected using a fluorescence microscope (magnification, $\mathrm{x} 200$ ) and western blotting. Subsequent experiments were performed $48 \mathrm{~h}$ following transfection.

Western blotting. Cell lysates were extracted using RIPA buffer (Beyotime Institute of Biotechnology) supplemented with a cocktail protease inhibitor (Roche Molecular Diagnostics), and the protein concentration was determined using a BCA protein assay kit (Beyotime Institute of Biotechnology) according to the manufacturer's protocol. A total of 5-40 $\mu \mathrm{g}$ cell total protein was separated by $10 \%$ SDS-PAGE. Proteins from macrophages treated with the Galectin-9-siRNA reagent were separated via $10 \%$ SDS-PAGE under nonreducing conditions. The proteins were then transferred onto PVDF membranes (GE Healthcare Life Sciences) by electroblotting. The membranes were blocked at $37^{\circ} \mathrm{C}$ for $1 \mathrm{~h}$ with $5 \%$ skimmed milk in Tris-buffered saline (TBS) with Tween-20 (0.1\%) and were then incubated for overnight at $4^{\circ} \mathrm{C}$ with antibodies against Galectin-9 (1:1,000; cat. no. AF2045, R\&D Systems, Inc.) and $\beta$-actin (1:1,000; cat. no. 4967, Cell Signaling Technology, Inc.). The membranes were washed with TBS washing buffer six times, and then incubated with horseradish peroxidase-conjugated goat anti-mouse (cat no. TA130001) or goat anti-rabbit (cat no. TA130015) secondary antibodies (1:2,000; OriGene Technologies, Inc.) at $37^{\circ} \mathrm{C}$ for $1 \mathrm{~h}$. Following further washing, the protein bands were visualized by Thermo Scientific Pierce ECL (cat. no. 32106, Thermo Fisher Scientific, Inc.) using an enhanced chemiluminescent system (Thermo Fisher Scientific, Inc.). The western blot images were quantified by optical density analysis (Image $\mathrm{J}$ version 1.8.0, National Institutes of Health).

Statistical analysis. All data and results were calculated from at least three replicate measurements and are presented as the 


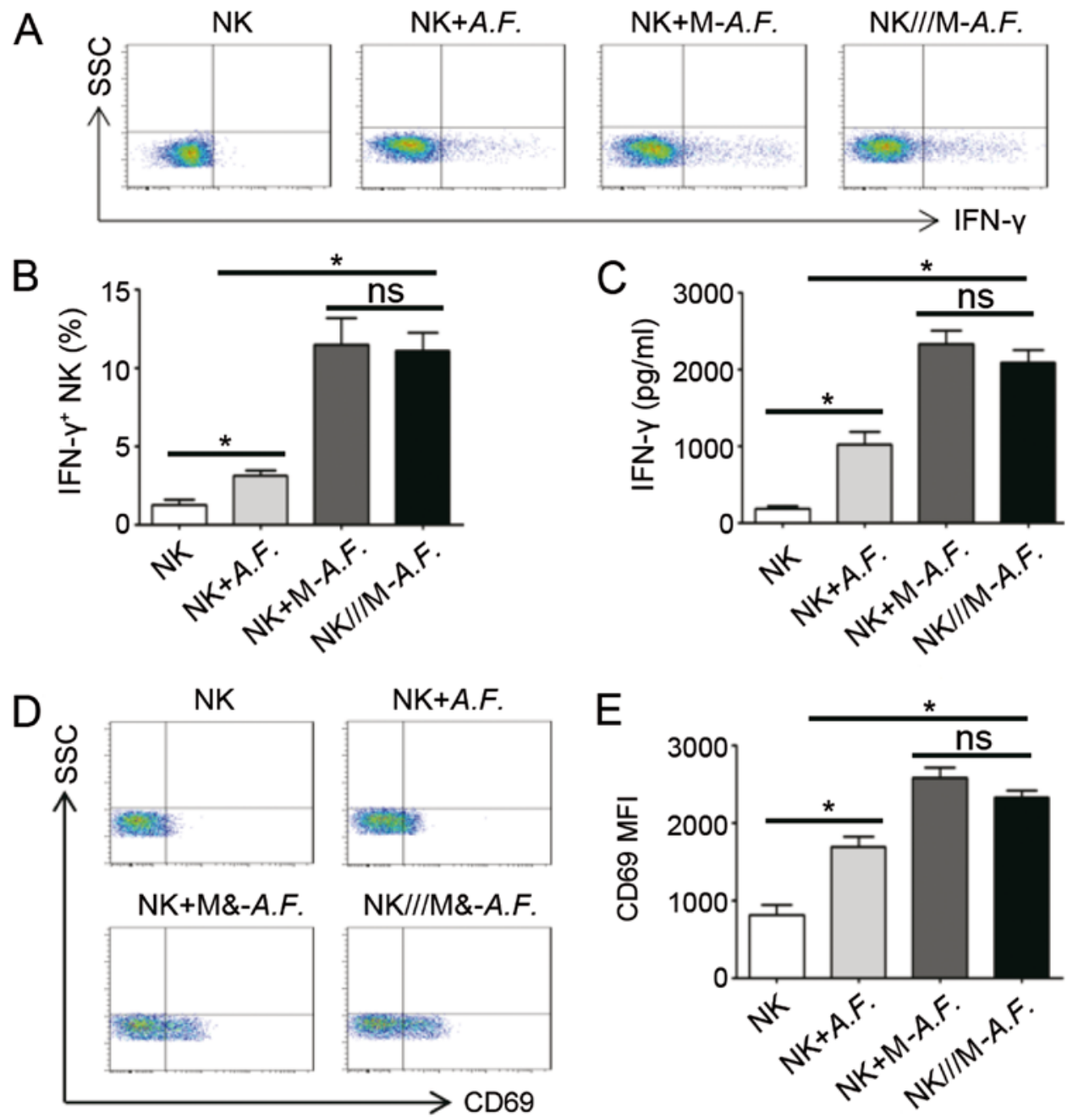

Figure 3. A.F. stimulates macrophages, which activate NK cells and upregulate IFN- $\gamma$ and CD69. Macrophages were stimulated with A.F. conidia (1:5) for $24 \mathrm{~h}$ and then co-cultured with NK cells from the same individual under various conditions for a further $12 \mathrm{~h}$. (A) IFN- $\gamma$ intracellular staining in NK cells. (B) IFN- $\gamma^{+}$NK cell percentage ( $n=3$ ). (C) ELISA analysis of the supernatants of the cell culture and co-cultures for IFN- $\gamma$ ( $n=3$ ). (D) CD69 expression on NK cells and (E) analysis of the MFI ( $\mathrm{n}=3)$. ${ }^{*} \mathrm{P}<0.05$, as indicated. A.F., Aspergillus fumigatus; $\mathrm{CD}$, cluster of differentiation; SSC, side scatter; MFI, median fluorescence intensity; interferon- $\gamma$, IFN- $\gamma$; M-, macrophage; NK cell, natural killer cell; ns, not significant; ///, separated by Transwell insert.

mean \pm standard deviation. Significant differences between experimental groups and the control group were determined using two-way analysis of variance followed by a Dunnett's t-test, mean values were compared using paired t-tests (two groups) followed by the Bonferroni correction for multiple comparison tests. using SPSS version 20.0 (IBM Corp.). $\mathrm{P}<0.05$ was considered to indicate a statistically significant difference.

\section{Results}

A. fumigatus induces macrophages to polarize into the MI type. Macrophages were cultured with activated A.fumigatus conidia for $24 \mathrm{~h}$, and the cells and conidia morphologies are presented in Fig. 1A. It was demonstrated that the swelling conidia of A.fumigatus significantly increased the expression of CD80 [Median Fluorescence Intensity, (MFI), 1,000 \pm 125 vs. $1,652 \pm 145 ; \mathrm{P}<0.05]$ and CD86 (MFI, 950 \pm 136 vs. $1,552 \pm 245 ; \mathrm{P}<0.05)$ in the macrophages (Fig. 1B). RT-qPCR analysis revealed that the mRNA expression levels of TNF- $\alpha$ $(\mathrm{P}<0.01)$, iNOS $(\mathrm{P}<0.05), \mathrm{CD} 80(\mathrm{P}<0.05)$ and CD86 $(\mathrm{P}<0.05)$ were significantly upregulated in the group treated with swelling conidia of the A.fumigatus compared with the control (Fig. 1C). The expression of the M2 type macrophages cell surface markers, CD163 and CD206, and IL-10 and arginase-1 gene expression were detected; however, there were no significant differences reported between the A.fumigatus-treated and control groups (data not shown). Collectively, the results suggested that $A$. fumigatus induced macrophages to polarize into the M1 phenotype.

A.fumigatus promotes M1 macrophages to secrete Galectin-9, TNF- $\alpha$ and IL-18. Galectin-9, TNF- $\alpha$, IL-18, IL-12 and IL-10 levels in the culture supernatants of macrophages treated with swelling conidia of A. fumigatus or control were detected via ELISA. The results revealed that $A$. fumigatus induced increased macrophage secretion of Galectin-9 (1,700 \pm 130 vs. $800 \pm 120 \mathrm{pg} / \mathrm{ml} ; \mathrm{P}<0.05), \mathrm{TNF}-\alpha(1,950 \pm 300 \mathrm{vs} .250 \pm 55 \mathrm{pg} / \mathrm{ml}$;

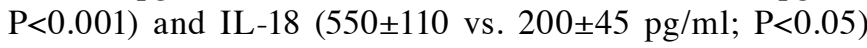
compared with the control; however, there were no significant differences in the concentrations of IL-12 or IL-10 (P>0.05; Fig. 2). 
A
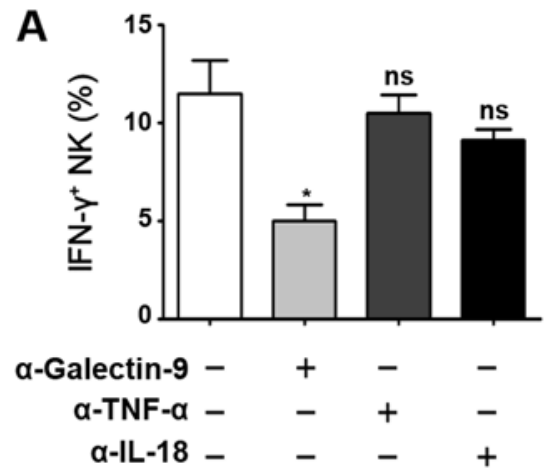

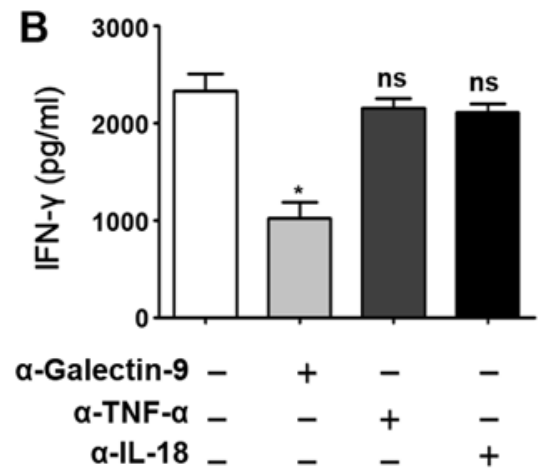

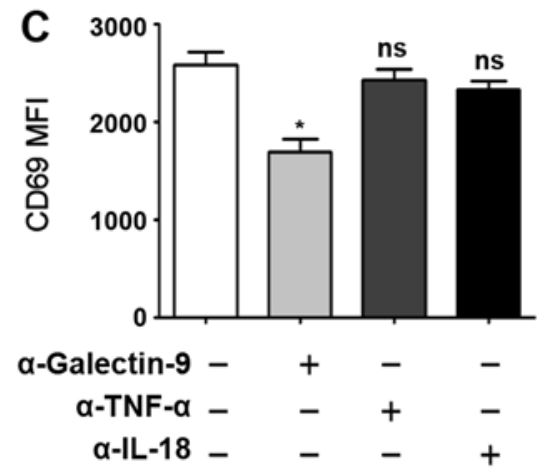

Figure 4. Galectin-9 is involved in the crosstalk between NK cells and macrophages in response to A.fumigatus. Macrophages were stimulated by A.fumigatus conidia (1:5) for $24 \mathrm{~h}$ and were then co-cultured with the NK cells from the same individual for a further $12 \mathrm{~h}$, in the presence or absence of Galectin-9-, IL-18- or TNF- $\alpha$-specific antibodies when added to NK cells. (A) IFN- $\gamma^{+}$NK cell percentage ( $n=3$ ). (B) IFN- $\gamma$ cytokine levels as determined by ELISA ( $\left.n=3\right)$. (C) CD69 expression on NK cells ( $n=3)$. ${ }^{*} \mathrm{P}<0.05$ vs. control. CD, cluster of differentiation; IL, interleukin; interferon- $\gamma$, IFN- $\gamma$; NK cell, natural killer cell; ns, not significant; TNF- $\alpha$, tumor necrosis factor- $\alpha$; A. fumigatus, Aspergillus fumigatus; MFI, median fluorescence intensity.

A. fumigatus activates macrophages, which then upregulate CD69 expression and IFN- $\gamma$ secretion in NK cells. Swelling conidia of A. fumigatus stimulated NK cells to secrete IFN- $\gamma$ Intracellular Staining (IC), $5.3 \pm 1.1$ vs. $1.1 \pm 0.4 \%$; ELISA, $1,050 \pm 100$ vs. $150 \pm 55 \mathrm{pg} / \mathrm{ml} ; \mathrm{P}<0.05)$ in the A. fumigatus and NK cell co-culture system. IFN- $\gamma$ expression was significantly increased when A. fumigatus-stimulated macrophages were added (IC, $5.3 \pm 1.1$ vs. $12.3 \pm 1.3 \%$; ELISA, $1,050 \pm 100$ vs. $2,450 \pm 300 \mathrm{pg} / \mathrm{ml} ; \mathrm{P}<0.05$; Fig. 3A-C). There was no significant difference in IFN- $\gamma$ expression in the NK cells when NK cells and monocytes were co-cultured in a Transwell setting ( $\mathrm{P}>0.05)$. The relative expression of CD69 on the NK cell surface between the various groups was similar to that of IFN- $\gamma$ (Fig. 3D and E). The results indicate that the interactions between the macrophages and NK cells were not dependent on cell-cell contact.

Macrophages treated with A. fumigatus regulate NK cells in a Galectin-9-dependent manner. To directly investigate the macrophage signals that induce NK cells to produce IFN- $\gamma$, antibodies against Galectin-9, TNF- $\alpha$ and IL-18 were added to the macrophage/NK cell co-cultures. The cell surface expression of CD69 and IFN- $\gamma$ levels were detected. It was observed that IFN- $\gamma$ secretion was significantly decreased following treatment with Galectin-9 antibody (IC, $12.3 \pm 1.3$ vs. $4.3 \pm 1.1 \%$; ELISA, $2,350 \pm 320$ vs. $1,040 \pm 120$ pg $/ \mathrm{ml} ; \mathrm{P}<0.05$; Fig. 4A and B). CD69 expression was also inhibited $(\mathrm{P}<0.05$; Fig. 4C); however, the TNF- $\alpha$ and IL-18 antibodies did not affect IFN- $\gamma$ or CD69 expression ( $\mathrm{P}>0.05$ ). To further demonstrate the important role of Galectin-9 in the macrophage activation of NK cells, cells were transfected with si-Galectin-9 to decrease the expression of Galectin-9 in macrophages. Western blotting was performed to detect the Galectin-9 silencing efficiency in the macrophages. As presented in Fig. 5A and B, Galectin-9 was significantly downregulated by $>50 \%$ in si-Galectin-9-transfected macrophages compared with the control. Additionally, the levels of Galectin- $9(\mathrm{P}<0.05)$ were decreased significantly in the si-Galectin-9-treated group supernatant compared with the control; however, the levels of TNF- $\alpha(\mathrm{P}>0.05)$ and IL-18 $(\mathrm{P}>0.05)$ were not affected (Fig. 5C). The number of IFN- $\gamma$-positive NK cells decreased significantly following Galectin-9 silencing in macrophages (Fig. 5D). Collectively, the results indicated that A. fumigatus induced macrophages to polarize into M1 macrophages by secreting Galectin-9, which then promoted NK cell activity and IFN- $\gamma$ secretion.

\section{Discussion}

A. fumigatus is a ubiquitous opportunistic fungus. The incidence of infection caused by A. fumigatus in immunocompromised patients has increased, and the mortality rate is at a high level (29). Following inhalation of A. fumigatus, the ciliated cells of the tracheal mucosa can remove A.fumigatus conidia automatically following indraft in a normal host; however, in an immunodeficient host, A. fumigatus conidia can colonize in the lungs and germinate into hyphae, eventually leading to the onset of invasive pulmonary aspergillosis (30).

Macrophages serve an important role in the elimination of invasive microorganisms, effectively capturing and removing A. fumigatus conidia by releasing acidified phagosomes and reactive oxygen intermediates (31). There are two major subtypes of macrophages, including the classically activated M1 macrophages and the alternatively activated M2 macrophages (22). M1 macrophages secrete proinflammatory cytokines, in contrast to M2 macrophages (23). The present findings revealed that the swelling conidia of A.fumigatus significantly increased the expression of CD80 and CD86 on macrophages, along with the expression of iNOS and TNF- $\alpha$. Additionally, the swelling conidia of Aspersions fumigatus promoted the secretion of Galectin-9, TNF- $\alpha$ and IL-18. TNF- $\alpha$ and IL-18 are two important Th1-type cytokines that exhibit strong immunomodulatory functions. They activate $\mathrm{T}$ and NK cells in vivo to contribute towards antitumor or anti-infection immune responses $(32,33)$.

The body's immune system is a net structure composed of a variety of immune cells (24). Therefore, the interaction between immune cells has received increasing attention. Activated macrophages interact with other immune cells via surface molecules (24). Intrahepatic macrophages reduce inflammatory injury and serve an antiviral role in the liver by interacting with NK cells (34). Monocytes regulate the functions of other 
A

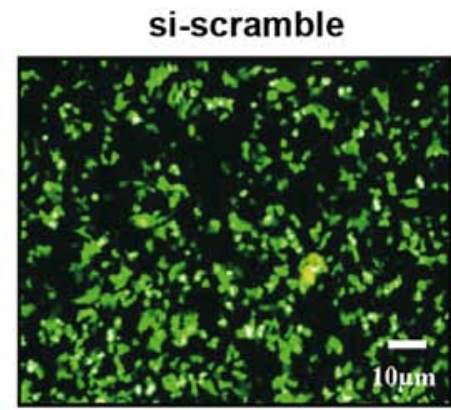

B

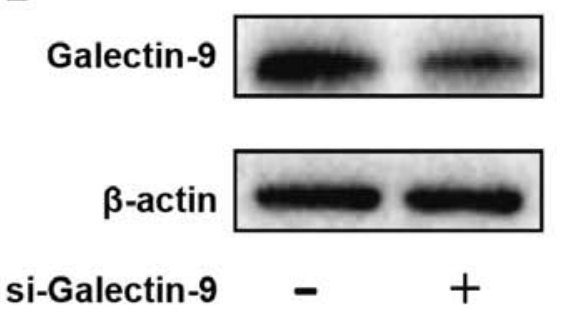

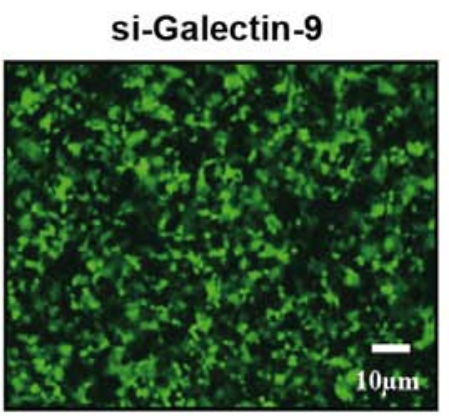

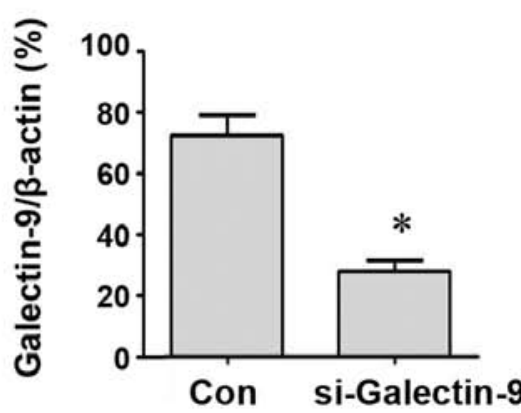

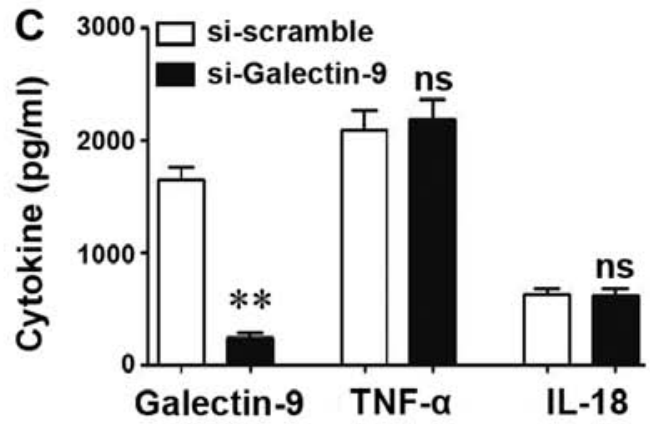

D

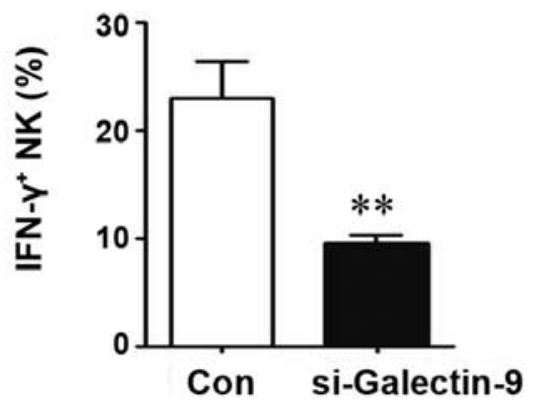

Figure 5. Galectin-9 silencing reduces the activation of NK cells induced by macrophages treated with Aspergillus fumigatus. (A) Monocyte-derived macrophages were transfected with si-Galectin-9 or scramble control. GFP was transfected to the cells together with siRNA treatment to display transfection efficiency. Scale bars, $10 \mu \mathrm{m}$. (B) Galectin-9 knockdown was demonstrated via western blot assay. ${ }^{*} \mathrm{P}<0.05$ vs. Con. (C) Galectin-9, IL-18 and TNF- $\alpha$ in the supernatants were detected by ELISA. ${ }^{* *} \mathrm{P}<0.01$ vs. si-scramble. (D) IFN- $\gamma+\mathrm{NK}$ cell percentage $(\mathrm{n}=3)$. ${ }^{* *} \mathrm{P}<0.01$ vs. Con. IL, interleukin; interferon- $\gamma$, IFN- $\gamma$; NK cell, natural killer cell; ns, not significant; si, small interfering RNA; TNF- $\alpha$, tumor necrosis factor- $\alpha$; Con, control.

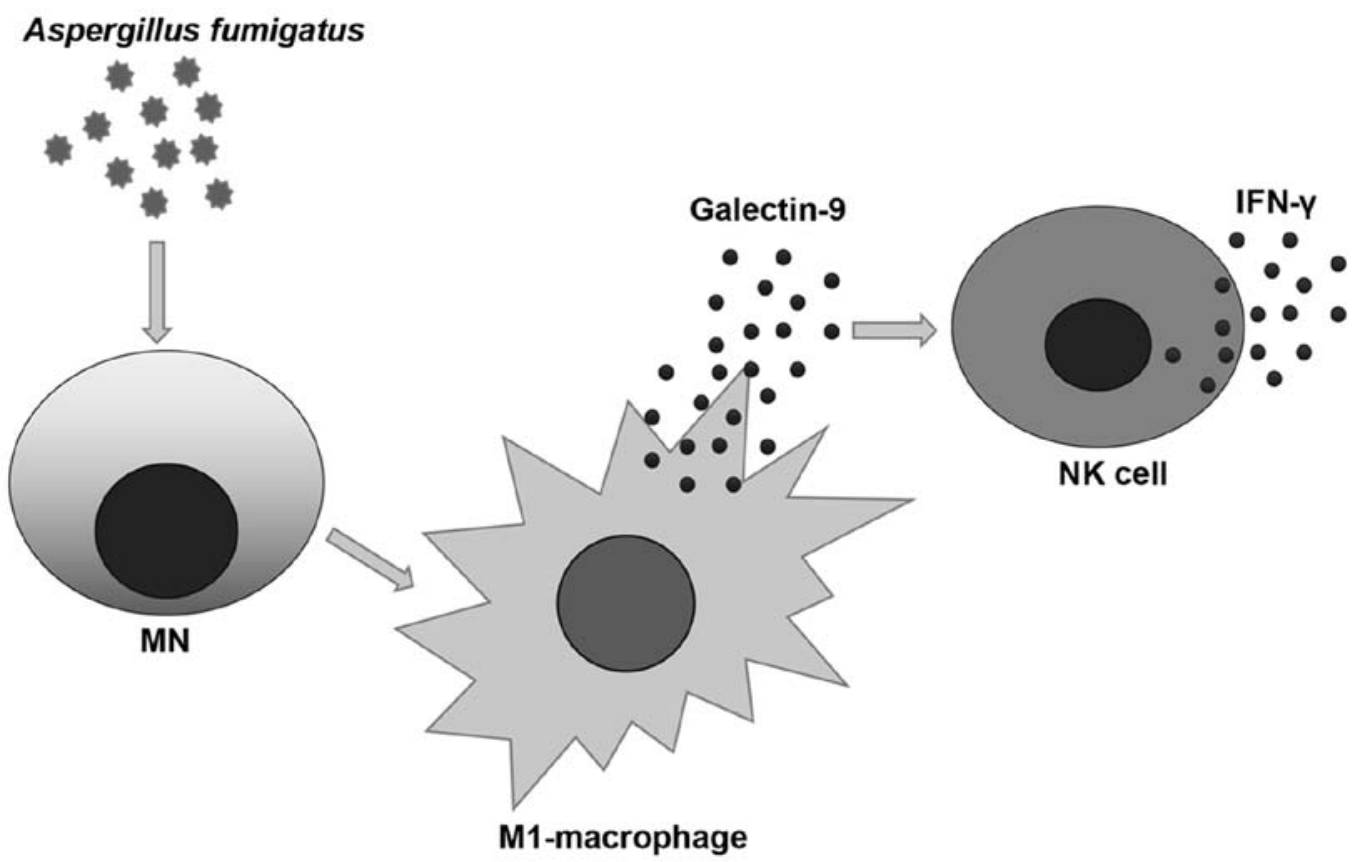

Figure 6. Schematic outline of A. fumigatus promoting human NK cell activity by regulating M1 macrophage polarization. A. fumigatus stimulated the polarization of MNs to M1 macrophages, leading to the secretion of galectin-9 and subsequent activation of NK cells, which then secrete IFN- $\gamma$. Interferon- $\gamma$, IFN- $\gamma$; MN, monocyte; NK, natural killer; A. fumigatus, Aspergillus fumigatus.

cells by secreting a variety of cytokines and expressing various surface proteins (10). The interaction between NK cells and monocytes is present in various tissues and organs, and peripheral blood (35); however, to the best of our knowledge, there has been limited investigation into the interaction between NK cells and monocytes in fungal infection. T-cell immunoglobulin and mucin-domain containing-3 (Tim-3) and 4-1BB are known receptors of Galectin- 9 on the surface of $\mathrm{T}$ cells and NK cells (35). Tim-3 was reported to act as a marker of activation or maturation of NK cells. Recombinant Galectin-9 enhanced the ability of NK cells to secrete cytokines and induce cytotoxicity in vitro (27). In the present study, it was observed that Galectin-9 
signaling regulated the function of NK cells following infection by Aspersions fumigatus; however, the effects of Galectin-9 signaling downstream molecules such as Tim-3 on NK cell function requires further investigation. Macrophage-derived Galectin-9 increased NK cell cytotoxicity in HCV infection, which may be associated with liver injury and persistent infection (24). It is important to understand whether there are interactions between natural immune cells, and to identify which key molecules serve an important role in the fungi and the host.

It has been reported that $A$. fumigatus activates NK cells, but the function of A.fumigatus in the interactions between macrophages and NK cells remains unclear (36). In the present study, it was observed that $A$. fumigatus conidia induced macrophages to polarize into M1 macrophages and secrete substantial quantities of Galectin-9. It was further revealed using antibody neutralization and gene silencing experiments that Galectin-9 served an important role in the interaction between macrophages and NK cells regulated by A. fumigatus. Collectively, a mechanism via which A.fumigatus polarized M1 macrophages by secreting Galectin-9 was identified, with downstream activation of NK cells and secretion of IFN- $\gamma$ (Fig. 6). These findings suggested a novel direction for the study of macrophages and NK cells in the inflammatory response to the A. fumigatus infection, and may aid the identification of potential strategies to control the progression of inflammation.

\section{Acknowledgements}

The authors would like to thank the staff of Jilin University Mycology Research Center for their assistance.

\section{Funding}

The present study was supported by the National Natural Science Foundation of China (grant no. 81271802) and the National Science and Technology Major Project of the Ministry of Science and Technology of China (grant no. 2013ZX10004612-006).

\section{Availability of data and materials}

The datasets used and/or analyzed during the current study are available from the corresponding author on reasonable request.

\section{Authors' contributions}

$\mathrm{XZ}$ performed the laboratory experiments and drafted the manuscript. DH analyzed the experimental data and revised the manuscript. SG and YW were involved in conducting the laboratory experiments. LW designed the project and supervised this study.

\section{Ethics approval and consent to participate}

The present study was approved by the Ethics Committee of Changchun Blood Center, Jilin, China and conducted in accordance with the approved guidelines for the 'Use of haemocytes in research'. Written informed consent for the use of haemocytes in research was obtained from all participants

\section{Patient consent for publication}

Not applicable.

\section{Competing interests}

The authors declare that they have no competing interest.

\section{References}

1. Dewi IMW, van de Veerdonk FL and Gresnigt MS: The multifaceted role of T-helper responses in host defense against Aspergillus fumigatus. J Fungi (Basel) 3: pii: E55, 2017.

2. Hellmann AM, Lother J, Wurster S, Lutz MB, Schmitt AL, Morton CO, Eyrich M, Czakai K, Einsele H and Loeffler J: Human and murine innate immune cell populations display common and distinct response patterns during their in vitro interaction with the pathogenic mold Aspergillus fumigatus. Front Immunol 8: 1716, 2017.

3. Schmidt S, Tramsen L, Schneider A, Schubert R, Balan A, Degistirici Ö, Meisel R and Lehrnbecher T: Impact of human mesenchymal stromal cells on antifungal host response against Aspergillus fumigatus. Oncotarget 8: 95495-95503, 2017.

4. Chakrabarti A, Chatterjee SS, Das A and Shivaprakash MR: Invasive aspergillosis in developing countries. Med Mycol 49 (Suppl 1): S35-S47, 2011.

5. Bao GQ, He L, Lee D, D'Angelo J and Wang HC: An ongoing search for potential targets and therapies for lethal sepsis. Mil Med Res 2: 20, 2015.

6. Osherov N: Interaction of the pathogenic mold Aspergillus fumigatus with lung epithelial cells. Front Microbiol 3: 346, 2012.

7. Hussell T and Bell TJ: Alveolar macrophages: Plasticity in a tissue-specific context. Nat Rev Immunol 14: 81-93, 2014.

8. Rosowski EE, Raffa N, Knox BP, Golenberg N, Keller NP and Huttenlocher A: Macrophages inhibit Aspergillus fumigatus germination and neutrophil-mediated fungal killing. PLoS Pathog 14: e1007229, 2018.

9. Sun H, Xu XY, Shao HT, Su X, Wu XD, Wang Q and Shi Y: Dectin-2 is predominately macrophage restricted and exhibits conspicuous expression during Aspergillus fumigatus invasion in human lung. Cell Immunol 284: 60-67, 2013.

10. Messlinger H, Sebald H, Heger L, Dudziak D, Bogdan C and Schleicher U: Monocyte-derived signals activate human natural killer cells in response to Leishmania parasites. Front Immunol 9: 24, 2018.

11. Rückerl D, Campbell SM, Duncan S, Sutherland TE, Jenkins SJ, Hewitson JP, Barr TA, Jackson-Jones LH, Maizels RM and Allen JE: Macrophage origin limits functional plasticity in helminth-bacterial co-infection. PLoS Pathog 13: e1006233, 2017.

12. Ewen EM, Pahl JHW, Miller M, Watzl C and Cerwenka A: KIR downregulation by IL-12/15/18 unleashes human NK cells from KIR/HLA-I inhibition and enhances killing of tumor cells. Eur J Immunol 48: 355-365, 2018.

13. Massawe R, Drabo L and Whalen M: Effects of pentachlorophenol and dichlorodiphenyltrichloroethane on secretion of interferon gamma (IFN $\gamma$ ) and tumor necrosis factor alpha (TNF $\alpha$ ) from human immune cells. Toxicol Mech Methods 27: 223-235, 2017.

14. Spörri R, Joller N, Albers U, Hilbi H and Oxenius A: MyD88-dependent IFN-gamma production by NK cells is key for control of Legionella pneumophila infection. J Immunol 176: 6162-6171, 2006.

15. Hansen DS, D'Ombrain MC and Schofield L: The role of leukocytes bearing natural killer complex receptors and killer immunoglobulin-like receptors in the immunology of malaria. Curr Opin Immunol 19: 416-423, 2007.

16. Ma LL, Wang CL, Neely GG, Epelman S, Krensky AM and Mody $\mathrm{CH}$ : NK cells use perforin rather than granulysin for anticryptococcal activity. J Immunol 173: 3357-3365, 2004.

17. Espinosa V, Dutta O, McElrath C, Du P, Chang YJ, Cicciarelli B, Pitler A, Whitehead I, Obar JJ, Durbin JE, et al: Type III interferon is a critical regulator of innate antifungal immunity. Sci Immunol 2: pii: eaan5357, 2017.

18. Morton CO, Bouzani M, Loeffler J and Rogers TR: Direct interaction studies between Aspergillus fumigatus and human immune cells; what have we learned about pathogenicity and host immunity? Front Microbiol 3: 413, 2012. 
19. Shi J, Zhao Y, Wang Y, Gao W, Ding J, Li P, Hu L and Shao F: Inflammatory caspases are innate immune receptors for intracellular LPS. Nature 514: 187-192, 2014.

20. Plebanek MP, Angeloni NL, Vinokour E, Li J, Henkin A, Martinez-Marin D, Filleur S, Bhowmick R, Henkin J, Miller SD, et al: Pre-metastatic cancer exosomes induce immune surveillance by patrolling monocytes at the metastatic niche. Nat Commun 8: 1319, 2017

21. Kochiyama T, Li X, Nakayama H, Kage M, Yamane Y, Takamori K, Iwabuchi $\mathrm{K}$ and Inada E: Effect of propofol on the production of inflammatory cytokines by human polarized macrophages. Mediators Inflamm 2019: 1919538, 2019.

22. Zhou D, Huang C, Lin Z, Zhan S, Kong L, Fang C and Li J: Macrophage polarization and function with emphasis on the evolving roles of coordinated regulation of cellular signaling pathways. Cell Signal 26: 192-197, 2014.

23. Zhang Q, Wang Y, Zhai N, Song H, Li H, Yang Y, Li T, Guo X, Chi B, Niu J, et al: $\mathrm{HCV}$ core protein inhibits polarization and activity of both M1 and M2 macrophages through the TLR2 signaling pathway. Sci Rep 6: 36160, 2016.

24. Li H, Zhai N, Wang Z, Song H, Yang Y, Cui A, Li T, Wang G, Niu J, Crispe IN, et al: Regulatory NK cells mediated between immunosuppressive monocytes and dysfunctional $\mathrm{T}$ cells in chronic HBV infection. Gut 67: 2035-2044, 2018.

25. Liang CC, Li CS, Weng IC, Chen HY, Lu HH, Huang CC and Liu FT: Galectin-9 is critical for mucosal adaptive immunity through the T Helper 17-IgA Axis. Am J Pathol 188: 1225-1235, 2018.

26. Golden-Mason L and Rosen HR: Galectin-9: Diverse roles in hepatic immune homeostasis and inflammation. Hepatology 66 : 271-279, 2017.

27. Nishio A, Tatsumi T, Nawa T, Suda T, Yoshioka T, Onishi Y, Aono S, Shigekawa M, Hikita H, Sakamori R, et al: CD14 ${ }^{+}$ monocyte-derived galectin-9 induces natural killer cell cytotoxicity in chronic hepatitis C. Hepatology 65: 18-31, 2017.
28. Livak KJ and Schmittgen TD: Analysis of relative gene expression data using real-time quantitative PCR and the 2(-Delta Delta C(T)) method. Methods 25: 402-408, 2001.

29. Gabrielli E, Fothergill AW, Brescini L, Sutton DA, Marchionni E, Orsetti E, Staffolani S, Castelli P, Gesuita R and Barchiesi F: Osteomyelitis caused by Aspergillus species: A review of 310 reported cases. Clin Microbiol Infect 20: 559-565, 2014.

30. Miceli MH and Kauffman CA: Aspergillus Galactomannan for diagnosing invasive aspergillosis. JAMA 318: 1175-1176, 2017.

31. Xu S and Shinohara ML: Tissue-resident macrophages in fungal infections. Front Immunol 8: 1798, 2017.

32. Timoteo RP, da Silva MV, Miguel CB, Silva DA, Catarino JD, Rodrigues Junior V, Sales-Campos H and Freire Oliveira CJ: Th1/Th17-related cytokines and chemokines and their implications in the pathogenesis of pemphigus vulgaris. Mediators Inflamm 2017: 7151285, 2017.

33. Kaplanski G: Interleukin-18: Biological properties and role in disease pathogenesis. Immunol Rev 281: 138-153, 2018.

34. Tu Z, Bozorgzadeh A, Pierce RH, Kurtis J, Crispe IN and Orloff MS: TLR-dependent cross talk between human Kupffer cells and NK cells. J Exp Med 205: 233-244, 2008.

35. Golden-Mason L, McMahan RH, Strong M, Reisdorph R, Mahaffey S, Palmer BE, Cheng L, Kulesza C, Hirashima M, Niki T and Rosen HR: Galectin-9 functionally impairs natural killer cells in humans and mice. J Virol 87: 4835-4845, 2013.

36. Bouzani M, Ok M, McCormick A, Ebel F, Kurzai O, Morton CO, Einsele $\mathrm{H}$ and Loeffler J: Human NK cells display important antifungal activity against Aspergillus fumigatus, which is directly mediated by IFN- $\gamma$ release. J Immunol 187: 1369-1376, 2011.

This work is licensed under a Creative Commons Attribution-NonCommercial-NoDerivatives 4.0 International (CC BY-NC-ND 4.0) License. 